\title{
Drones and windbreaks
}

\author{
Petru Cardei ${ }^{1, *}$, Sebastian Muraru ${ }^{1}$, and Mihai Matache ${ }^{2}$ \\ ${ }^{1}$ INMA Bucharest, Computerized Engineering Department, 013813 Bucharest, Romania \\ ${ }^{2}$ INMA Bucharest, Testing Department, 013813 Bucharest, Romania
}

\begin{abstract}
The article presents a possibility of applying the techniques specific to the drones (UAV) in order to estimate the efficiency, and optimize the windbreaks (vegetative barriers, forest curtains).In the first part the article presents a variant of determination of the dynamic and optical porosities of forest protection curtains using one or two drones simultaneously. In the second part, ascertaining some language inaccuracies, it observes that the issue of the efficiency of the forest curtains became a problem of mitigation of wind intensity. Finally, the last proposed application shows that using the drones, you can estimate the protected volumes behind the curtains, basically giving up the optical porosity. In order to achieve these applications, there is an essential condition: records of the magnitude of the wind speed and of the coordinates must not be excessively affected by the wind.
\end{abstract}

\section{Introduction}

The definition of the windbreak will be accepted after [1] or [2] (row of trees or a fence, wall, or screen, which provides shelter or protection from the wind). Synonymous terms, as used in this article, are vegetative barriers (usually formed by high herbs) or forest curtains or shelterbelt. We will not detail the definition of the windbreak, but will take over the definition and we will summarize the information from [2]. A windbreak (shelterbelt) is a planting usually made up of one or more rows of trees or shrubs planted in such a manner as to provide shelter from the wind and to protect soil from erosion. Other benefits include contributing to a microclimate around crops, providing habitat for wildlife, and, in some regions, providing wood if the trees are harvested, [4]. The importance of vegetal barriers is also highlighted in the case of the protection of communication ways, [5].

We followed the path described by the authors [3] until to the method of obtaining the experimental data. Vegetative barrier effectiveness is commonly measured by its sheltering efficiency leeward of the barrier via reduced wind speed, [3].The aerodynamic $\left(\alpha_{p}\right)$ and the optical $\left(\beta_{p}\right)$ porosities of the vegetative barrier are defined in [3]. The aerodynamic porosity is defined by the formula:

$$
\alpha=\frac{\int_{0}^{H} \bar{v} d z}{\int_{0}^{H} \bar{v}_{0} d z}
$$

\footnotetext{
* Corresponding author: petru cardei@yahoo.com
} 
where $H$ is the height of the barrier, in $\mathrm{m}, \bar{v}$ is the leeward wind speed (bleed wind speed), in $\mathrm{m} \mathrm{s}^{-1}$, and $v_{0}$ is the mean windward wind speed, in $\mathrm{m} \mathrm{s}^{-1}$. The optical porosity is defined by the formula, [3]:

$$
\beta=\frac{(\text { Background area })}{(\text { Total area })}
$$

According to [3], the background area (representing gaps outside the area occupied by the tree including leaves, branches, stems, and trunk), is obtained from image analysis of digitized photographs. Although $\beta_{p}$ is convenient to use because it does not require wind measurement, a relationship between $\alpha_{p}$ and $\beta_{p}$ is necessary, especially for deciduous trees that experience porosity variations throughout the year, as it is specified in [3].

Specific objectives of the study from article [3], were to: determine optical porosity using image analysis, determine aerodynamic porosity and drag coefficients during leaf-on and leaf-off conditions using wind profile measurements, and measure the effects of vegetative barrier leaf condition on wind speed reduction.

This article proposes a new approach, with new tools and concepts, for the same problem of forest curtains. Using the drones to measure parameters and more accurately defining a wind-dampening factor were the main objectives of this study.

\section{Material and methods}

For the data acquisition experiments, as in [3], it is primarily necessary to have an area containing vegetal barriers, with the properties required in [3]. At the beginning of the proposed activity, it is advisable to take into account the indications from [3], the site must be chosen based on the following criteria: (a) flat terrain to avoid topographical effects on wind speed patterns close to the vegetative barrier; (b) absence of obstructions such as buildings near the vicinity to avoid adverse effects on wind speed patterns along the vegetative barriers; (c) similar vegetation height on both sides of the vegetative barrier to maintain constant surface roughness; and (d) minimum length : height ratio of 10:1 to avoid edge and vortex effects at the ends of the vegetative barrier.

The description of the geographic areas chosen for experimentation, the climatic conditions and the vegetation type will be made when the experiments will be performed and the results will be presented, as in [3].

\subsection{Wind Profiles}

This is the first step of the method, which will be different from [3]. To determine the wind speed profiles, the authors [3] used two towers on which they placed several anemometers. In the experiences which are proposed in this article, one or more drones will be used. For measuring wind speed, drones (UAVs) will be equipped with anemometric sensors. These sensors will measure the speed and direction of the wind. Such sensors, for drone, exist in commerce, for example [6], [7], [8] and [9].

\subsection{Porosity determination and vegetative barrier drag coefficient}

To determine optical porosity, the same methods as [3] can be used, but using images obtained with a proper camera, installed on the drone (UAV). The linear force density along the forest curtain and the drag coefficient, can be calculated according to formula (3) and (4) from [3], neglecting the atmospheric pressure in the two parts of the curtain: 


$$
D_{b}=\int_{0}^{z}\left[\left(\frac{1}{2} \rho_{a i r} u_{u p}^{2}\right)-\left(\frac{1}{2} \rho_{a i r} u_{d o w n}^{2}\right)\right] d z, C_{D}=\frac{2 D_{b}}{\left(\rho_{\text {air }} \bar{u}^{2} H\right)}
$$

\subsection{Meteorological data and data analysis}

As in [2], one meteorological station will be used on board each of the drones used. These stations will record: atmospheric temperature, humidity and pressure. All parameters will be reported to the geographic coordinates. In this paper, we are only predicting the structure of the data because we have not yet done such experiments with drones in the vicinity of the windbreaks. The data recorded during the flight of the drones in the neighbourhood of the windbreaks will contain the following parameters:

\begin{tabular}{|c|c|c|c|c|}
\hline No. & Data & Parameters & Notation & Units \\
\hline \multirow[t]{5}{*}{1} & Geographic & Latitude & $\lambda$ & degree \\
\hline & Coordinates & Longitude & $\theta$ & degree \\
\hline & & Altitude & $h$ & $\mathrm{~m}$ \\
\hline & & Local abscissa & $x$ & $\mathrm{~m}$ \\
\hline & & Local coordinate & $y$ & $\mathrm{~m}$ \\
\hline \multirow[t]{5}{*}{2} & Meteorological data & Atmospheric temperature & $T$ & Celsius degree \\
\hline & & Atmospheric pressure & $p$ & $\mathrm{~Pa}$ \\
\hline & & Humidity in the atmosphere & $u$ & $\%$ \\
\hline & & Average $^{\dagger}$ wind speed & $v$ & $\mathrm{~ms}^{-1}$ \\
\hline & & $\begin{array}{l}\text { Direction of the maximum wind } \\
\text { speed }\end{array}$ & $v$ & grade \\
\hline 3 & Photographic images & Windbreaks images & If & - \\
\hline 4 & Local time & & $t$ & $\begin{array}{l}\text { Hours, } \\
\text { sec }\end{array}$ \\
\hline
\end{tabular}

All data from the Table 1 will be recorded simultaneously in the designated data acquisition locations.

\subsection{Setting up the drones route and storing data}

To make the experiments as effective as possible, it's a good idea to set the drones' route before the start of the experiments. Also, the data sampling locations will be pointed out. The software will specify the sampling points of the data that will be automatically stored. An equidistant point network is recommended at varying distances $(0.5$ to 30 times the windbreak height of the longitudinal axis of the windbreak and at set heights up to 0 to 6 times the height of the windbreak). The record list will take the form:

$$
\left\{\lambda_{i}, \theta_{i}, h_{i}, T_{i}, p_{i}, u_{i}, v_{i}, v_{i}, I f_{i}, t_{i}\right\}, i=1, \ldots, N
$$

where $N$ is the number of recordings made. The geographic coordinate array can be converted to local Cartesian coordinates using the national coordinate system (e.g. STEREO70 or WGS84). Is obtain the next string: $\left\{x_{i}, y_{i}, z_{i}, T_{i}, p_{i}, u_{i}, v_{i}, v_{i}, I f_{i}, t_{i}\right\} ; i=1, \ldots, N$, and the conversion errors are negligible for the purposes (assuming $z_{i}=h_{i}$ ).

Therefore, in the data processing process, the following points of the fields (time-space dependent parameters) are obtained: maximum speed $\left\{v_{i}, v_{i}, \lambda_{i}, \theta_{i}, h_{i}, t_{i}\right\} ; i=1, \ldots, N$, or in

\footnotetext{
$\dagger$ When is working with a special anemometer (e.g. multi-parametric sonic anemometer, [9], [17], one can measure the three components of the wind velocity at a given time.
} 
Cartesian coordinates $\left\{v_{i}, v_{i}, x_{i}, y_{i}, z_{i}, t_{i}\right\} ; i=1, \ldots, N$, and the pressure, $\left\{p_{i}, \lambda_{i}, \theta_{i}, h_{i}, t_{i}\right\} ; i=$ $1, \ldots, N$ and in Cartesian coordinates $\left\{p_{i}, x_{i}, y_{i}, z_{i}, t_{i}\right\} ; i=1, \ldots, N$ and similarly for the humidity and atmospheric temperature field, but these fields do not directly interest in the first stage of calculation. There is also an important field of computation, namely that of the photographic images, which are processed, and generates, according to formula (2), a field of optical porosity (variable in space and time): $\left\{\beta_{i} x_{i}, y_{i}, z_{i}, t_{i}\right\} ; i=1, \ldots, N$ is generated. Operating with two drones simultaneously and collecting the data, on the each part of the forest curtain, in symmetrical network points in report to the axis of the windbreak, the next vectors of data will be obtained:

$$
\left\{\lambda_{0 i}, \theta_{0 i}, h_{0 i}, T_{0 i}, p_{0 i}, u_{0 i}, v_{0 i}, v_{0 i}, I f_{0 i}, t_{0 i}\right\} ; i=1, \ldots, N
$$

for the semi-space in which the wind enters the windbreaks and (4) for the semi-space where the wind comes out of the windbreaks. In this case, using the formula (1), and eventually a process of interpolation of the maximum speed field, an aerodynamic porosity field is obtained. Next, this field is averaged in a volume area given on the each side of the windbreaks. It can also proceed by calculating the average velocity and then directly calculating the aerodynamic porosity (1).

When working with at least two drones at the same time, each of them on different part of the forest curtain in the vectors (4) and (12) will be satisfied (by the working mode, or even by interpolation), the equality: $t_{i}=t_{0 i}$. If the work is not done with two drones simultaneously, but only one, then you have to use the hypothesis that the wind has not undergone significant direction and intensity changes during the data collection process (which is physically unlikely in many cases).

\subsection{Data processing}

Data stored in the forms (4) and (5) can be processed to obtain aerodynamic and optical porosities. Data processing can be done in the same way as in [3], but it can also be used in a more complex way to processing. This option will briefly describes in order to be prepared for then when the data will be available.

Data is collected in the form (4) or (4) and (5) and then, by interpolation, the next functions will be obtained: $v, v_{0}, u, u_{0}, v, v_{0}, p, p_{0}, T, T_{0}$ in geographic or, in local Cartesian coordinates, $x, y, z, t$.

Using the definitions of the wind velocity field (vector) can be defined in this way:

$$
\vec{v}(t)=v_{x}(t) \overrightarrow{l_{x}}+v_{y}(t) \overrightarrow{l_{y}}+v_{z}(t) \overrightarrow{l_{z}}
$$

atmospheric pressure and temperature being scalar fields. The direction of maximum wind speed is established using the wind speed in Cartesian coordinates. The wind speed fields from the front and behind the windbreak has to be inserted as:

$$
\begin{gathered}
v_{w x}=v_{x}(x, y, z, t), v_{w y}=v_{w y}(x, y, z, t), v_{w z}=v_{w z}(x, y, z, t), \text { for } y>\varepsilon \\
v_{l x}=v_{x}(x, y, z, t), v_{l y}=v_{y}(x, y, z, t), v_{l z}=v_{z}(x, y, z, t), \text { for } y<-\varepsilon
\end{gathered}
$$

and in the same manner, for the other parameters: $u_{w}, p_{w}, T_{w}, u_{l}, p_{l}, T_{l}$, where $\varepsilon$ is a constant of the windbreak, equal to, for example, the distance at which the drone can safely approach the curtain axis (due to the crown of the trees). The constant $\varepsilon$ is also the limit of approach of the drones to the axis of the windbreak, so that the parameters fields will not measure in the intense turbulence area. The temporal mean of the physical parameters from (7), over a given time interval of length $\Theta$, is defined by (8): 


$$
\bar{v}_{w x}=\frac{1}{\Theta} \int_{0}^{\Theta} v_{x}(x, y, z, t) d t, y \in[\varepsilon, n H]
$$

and in the same manner for the other parameters, see [18]. Similarly, spatial average is defined by integrating the physical fields involved in the process, on the considered atmospheric volumes, using the triple integral, see [18].

\subsection{Aerodynamic porosity}

Dynamic porosity, as defined in [3], and in this paper in (1), is interpretable. The two speeds involved in the calculation formula (1), are defined as average speeds, but a way to calculate these mean values is not defined. Wind speed is a vector field that depends on the temporal variable and has three components, (15).Be:

$$
v=\sqrt{v_{x}^{2}+v_{y}^{2}+v_{z}^{2}}
$$

the intensity of wind speed. Be $\theta$ the angle between the $O x$ axis and the projection of the vector velocity on the plane $x O y$, and $\beta$ the angle between the wind speed vector, and its projection on the plane $x O y$. Using these notations, the components of the velocity vector can be written as:

$$
v_{x}=v \cos \beta \cos \theta, v_{y}=v \cos \beta \sin \theta, v_{z}=v \sin \beta
$$

The system of equations (10) connects the two manners to give the wind velocity vector: by coordinates, respectively, by velocity and angular coordinates (polar coordinates). Expressing the intensity of the velocity, as a function of the angular polar coordinates, and by annulling the derivatives, the directions of maximum wind intensity is obtained:

$$
\theta=\operatorname{arctg} \frac{v_{y}}{v_{x}}, \beta=\operatorname{arctg} \frac{v_{z}}{\sqrt{v_{x}^{2}+v_{y}^{2}}}, \theta \in\left[-\frac{\pi}{2}, \frac{\pi}{2}\right], \beta \in[0, \pi] .
$$

Therefore, using the relations (11), it is possible to switch from the wind sensor information that gives the velocity vector through the Cartesian components to the information that gives the velocity vector through the maximum speed value. Next, the problem that needs to be clarified is that of estimating the effect of attenuation of the wind intensity produced by the forest curtains. Since the average velocity value can be done in time and space, a calculation method applicable to the wind speed field must be established both in front and behind the protection curtain and for any type of protection (having the axis, line right or curve, right line on portions, single tree line or more, possibly from several species). For example, we the average time speed of the wind can be defined, like in [10]:

$$
\bar{v}=\bar{v}(x, y, z, t)=\frac{1}{T} \int_{t}^{t+T} v(x, y, z, t) d t, T>0
$$

If the estimation of efficiency is done on a finite length of the forest curtain, then, for the calculus of the aerodynamic porosity, the average spatial value of the temporal mean value can be proposed:

$$
\widetilde{v_{f}}=\frac{1}{L(h-\varepsilon) H} \int_{0}^{L} \int_{\varepsilon}^{h} \int_{0}^{H} \bar{v}(x, y, z, t) d x d y d z
$$

and in the same manner, with changing of the integral limits after $O y$ axis for the volume in front of the windbreak. Thus, a measure of the aerodynamic porosity of the windbreak can be defined: 


$$
\alpha=\frac{\widetilde{v_{b}}}{\widetilde{v_{f}}}
$$

The ratio (14) is rather a measure of the wind intensity attenuation by the forest curtain. Conventionally we admit that this ratio can be a measure of the dynamic porosity of the forest curtain. In terms of spatial mediation, it should be noted that the mean values (13) are calculated on parallelepiped volumes only when studying areas of small length of a windbreak. In the case of estimating the efficiency of a windbreak over its entire length, the average velocity in front of the windbreak can maintain formula (13), but for the average velocity behind the windbreak, the integration volume changes, being less than that considered in the above considerations. In order to understand the volume delimitation mode on which the average wind speed value is calculated behind the windbreak, the readers are sent to [11].

\subsection{Mode of operation in the field}

According to the issues problems discussed from [3], the working process of the drones should aim at measuring the aerodynamic porosity and the optical porosity of the windbreaks. The working process may only include determining the wind speed field if the objective is to calculate the efficiency, and the optical porosity estimation is not necessary. By computing, as in other trials, a relationship will be established between these two porosities. The relationship between the two porosities may depend on the season, see [18], wind speed, type of the trees, atmospheric humidity. These dependencies can only be determined by repeated measurements under different environmental conditions. To make accurate measurements, it is recommended to operate with two drones, each doing the same type of measurements simultaneously, on different parts of the windbreak.

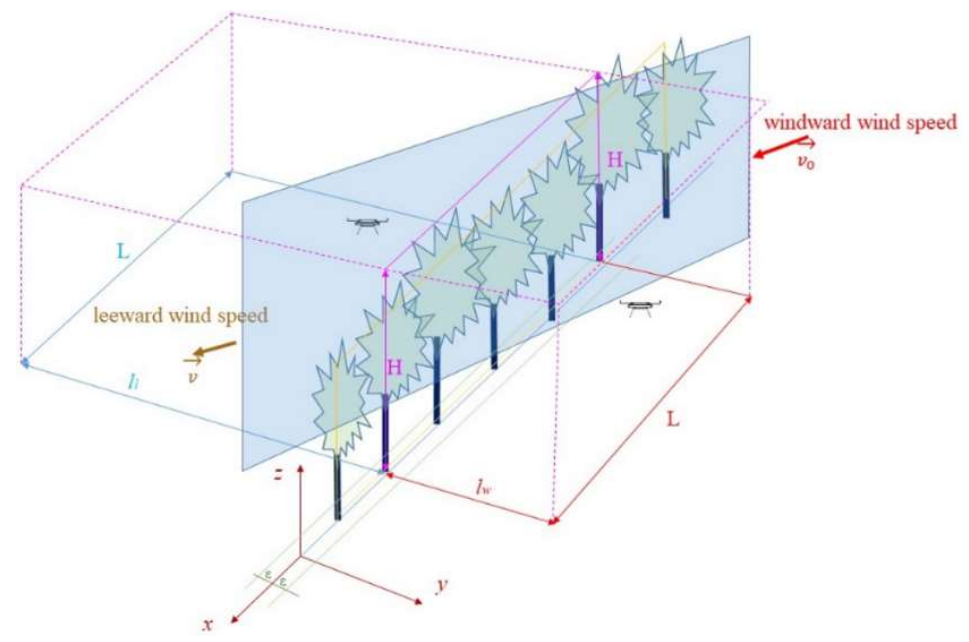

Fig. 1 The schema of the process of estimating the efficiency of a windbreak, using two drones simultaneously.

When working with two drones, one on each side of the forest curtain, they will sweep the chosen test volumes: $[0, L] \times[\varepsilon, h] \times[1.5,2 H]$, in the front of the windbreak, respectively $[0, L] \times[-h,-\varepsilon] \times[1.5,2 H]$, behind the forest curtain, see Figure 1 . Lower and upper vertical limits are conventionally chosen by the operator team to cover the area of interest. Also, the constant $\varepsilon$, will be chosen so that no measurements are made in the area of intense turbulence near the windbreak. Depending on the experimental availability, the depth 
of the domain, $h$, can be chosen up to $10 \mathrm{H}-20 \mathrm{H}$, [3]. The mode of operation of each of the two drones consists in stabilizing it in a measuring location for a minimum time interval to achieve wind speed recording and mediation. The following data vector is recorded in each measuring position:

$$
\left(x_{i}, y_{i}, z_{i}, \bar{v}_{x i}, \bar{v}_{y i}, \bar{v}_{z i}, t_{i}, \operatorname{Im}_{i}\right)
$$

where $z_{i}>\varepsilon$ for the drone which is working in front of the windbreak, respectively $z_{i}<-\varepsilon$, for the drones which working behind the windbreak. The symbol $I m_{i}$ is the image taken by the video camera of the drone, at the record with the index $i$.

If you are only interested in the intensity of the wind and possibly the direction of maximum intensity, you can move from the data structure (9) to the data structure (10):

$$
\left(x_{i}, y_{i}, z_{i}, \bar{v}_{i}, \bar{\theta}_{i}, \bar{\beta}_{i}, t_{i}, I_{i}\right)
$$

using the Cartesian transformation and the formulas, (10), (11). The index $i$, varies from 1 to $N_{f}$, in front of the windbreak and from 1 to $N_{b}$ behind the windbreak. The average wind speed values, which appear in (15) and (16) are calculated using the formula:

$$
\bar{v}_{i}=\frac{1}{T} \sum_{j=1}^{M} \frac{v_{i j+1}+v_{i j}}{2}\left(t_{j+1}-t_{j}\right), 0=t_{1}<t_{2}<\cdots<t_{M+1}=T .
$$

where $v_{i j}$, is the speed recorded in the location $i$ at the time $t_{j}$. If the recording time step at each location is constant, then the average (17) becomes the arithmetic average. For the other average data components measured, the formulas are similar, see [18].

In order to make a correct evaluation of the dynamic porosity, the reference speeds (6) - (7), averaged in relation to the spatial coordinates and thus located in the centre of the considered parallelepiped volume, should be reported. We can accept the hypothesis that in front of the forest curtain, the wind has small variations in relation to those behind the windbreak, so that it will be considered $N_{f}<<N_{b}$. This means that the fluctuations in relation to the spatial coordinates are small in front of the windbreak in relation to the fluctuations of the wind speed behind the windbreak, without a narrow zone located along the forest curtain. For a first approximation of spatial mediation, one can consider the following arithmetic averages:

$$
\hat{v}_{f}=\frac{\sum_{1}^{N_{f}} \bar{v}_{i}}{N_{f}}, \hat{v}_{b}=\frac{\sum_{1}^{N_{b}} \bar{v}_{i}}{N_{b}}
$$

the first sum contain all the temporal average values of the maximum wind speed measured by the drones sensors in the front of the windbreak, and the second sum containing all timeaveraged speeds determined by the drones behind the windbreak. A more precise variant of the spatial average can be obtained if there is availability of a drone's action plan, so that the spatial volumes in front and behind the windbreaks include at least 27 measurement points, in Cartesian network in each volume. In this case, the formulas (13), approximated by Riemann sums, according to the theory of the triple integral, for example [12], can be used for spatial average. Another possible option for spatial average of the wind speed calculus is the polynomial interpolation of experimental data, or other type of interpolation of temporalspan speeds, followed by the analytical exact calculus, using the formula (13).

\section{Effectiveness of forest curtains - a mitigation problem}

Since, generally, behind the windbreak, at a certain distance and height, the wind speed returns to the value of the one before the windbreak, the measure (14) of the forest curtain 
efficiency, cannot be correct unless it specifies the distance and the height at which it is made determining the wind speed behind the windbreak. The efficiency of the windbreaks can also be thought in other terms. In the design of vegetal barriers, the optimal height of their operation and the maximum limit of the attenuated speed, depending on the objective or the protected objectives, are specified, [5], [13].

In [14] there are results on the wind intensity behind the forest curtain at different distances and heights. Using Figure 6 from [14], we obtained data required to be able to estimate the distribution of wind intensity and to set a criterion for the efficiency of the barrier. To express the effectiveness of the forest curtain as a non-dimensional parameter, it is possible to divide the measured efficiency by the wind velocity return distance to the average value in front of the forest curtain, through the height of the curtain. We obtain the expression of a dimensionless efficiency, which can also be expressed in percentages: $f_{a}(h)=\delta(h) / H$ in which the distance $\delta(h)$, is the one at which the wind intensity, behind the windbreak, at height $h$ (to the ground), is equal to the average wind speed in front of the forest curtain. Conventionally, $\delta$ can be a distance to the windbreak, for which the average speed behind the windbreak reaches the average value from the front of the same windbreak (or a fraction thereof) at any height, $h, \delta=\delta(h)=k H$. In this case, obviously, $f_{a}=k$.

Another way of defining the factor of attenuation of the wind intensity for a forest curtain is inspired by the graphical representation of the wind speed increase behind the windbreak [14]. It is possible to define the attenuation factor of the forest curtain, starting from the wind velocity distribution behind the curtain, in relation to the intensity of the wind speed in front of the curtain (considered as a mean constant value over time, but depending on spatial coordinates). Either the velocity field behind the curtain (Figure 1):

$$
v_{b}=v_{b}(x, y, z), y<-\varepsilon,
$$

It is considered a narrow section of the forest curtain so that the intensity variation (31) is negligible in relation to the variable across the windbreak, $x$ (Figure 1). The above considerations also lead to more complex ways of defining the wind speed attenuation by a windbreak. Suppose, for example, using a drone, the time-averaged speed, more precisely the mean time value of the vector wind velocity is measured at some points behind the curtain. Because there aren't any own experimental data, the experimental data displayed as graphics is taken from [15]. Using the data published in [15] for an orange windbreak for an average wind speed in front of the $7 \mathrm{~m} / \mathrm{s}$ barrier and an average barrier height $8.2 \mathrm{~m}$, some estimation of the wind speed distribution behind the windbreak can be obtained.

Using the experimental information from [15], a distribution of the velocity field can be given in a plane section perpendicular to the axis or line of the forest curtain. Distribution can be obtained by interpolation. Such a distribution can be obtained by collecting the intensity of the wind speed in several locations behind the forest curtain. Using a wind distribution map, we can formulate different type of estimators of mitigate of wind intensity in the presence of a windbreak. The attenuation factor is defined as the ratio between the area of the field in which the velocity is less than the given speed and the total area of the same domain. If the maximum allowable speed is defined as a percentage of the average wind speed in front of the windbreak, then the maximum allowable speed is: $v_{a}=k_{a} v_{f}$. Maps of the velocity distribution in the atmospheric spaces in front and behind the vegetal barrier, can also be obtained by numerical simulation, see [18]. For comparison with the maps given in [18], [16] can be consulted, for example.

Starting from the surface definition of a plan area using Darboux sums, we calculate the area of the domain where the wind speed is below the maximum allowable speed:

$$
A_{u}=\sum_{i=0}^{n_{y}-1} \sum_{j=0}^{n_{z}-1}\left(y_{i+1}-y_{i}\right)\left(z_{j+1}-z_{j}\right) \theta\left(w_{i, j}\right)
$$


where:

$$
\theta\left(w_{i, j}\right)=\left\{\begin{array}{l}
1, \text { if } w_{i, j} \leq k_{a} v_{f} \\
0, \text { if } w_{i, j}>k_{a} v_{f}
\end{array}\right.
$$

In (20) and (21),

$$
w_{i, j}=v\left(x_{s}, y_{i}, z_{j}\right), i=0, \ldots, n_{y}, j=0, \ldots, n_{z}
$$

The coordinate along the windbreak line, $x_{s}$, is fixed, and $\left\{\left(y_{i}\right)_{i=1, \ldots, n_{y}}\right\},\left\{\left(z_{j}\right)_{j=1, \ldots, n_{z}}\right\}$, are meshing of the edges of the volume considered, along the $O y$ axis, respectively $O z$. The function $v$ from (22) is obtained by interpolation from data collected by the drone. For the total area of the domain the dimensions of the rectangle can be directly used, or the formula:

$$
A_{T}=\sum_{i=0}^{n_{y}-1} \sum_{j=0}^{n_{z}-1}\left(y_{i+1}-y_{i}\right)\left(z_{j+1}-z_{j}\right)
$$

With these data, the attenuation factor will have the expression: $f_{a}=A_{u} / A_{T}$. For the dimensions of the section by $10 \times 300 \mathrm{~m}$ and the characteristics of the case exposed in [15], taking $k_{a}=0.95, f_{a}=0.679$ is obtained. It can be seen that this attenuation factor depends on the length of the domain considered and its height. It also depends on the state of the crown of the windbreak and its type. In the formula (20), factors that take into account the sizes of the field used for agricultural crops can be introduced. For example, if the calculation is limited to a distance of $200 \mathrm{~m}$ behind the windbreak, the attenuation factor increases to 0.757. The limitation can also be made in height. The statement that the agricultural crop behind the windbreak needs protection up to a height of $3 \mathrm{~m}$ has been supposed. In this case the domain is restricted to this height (we keep the domain length is kept at $200 \mathrm{~m}$ ), and, perhaps contrary to the expectations, the attenuation factor is lower than in the previous case, its value being 0.683 . The explanation for this result can be found in the structure of the windbreak in the area of the stems, where, among the trunks of the trees, a wind of less attenuated intensity enters. In the case of complex windbreaks with double or triple rows of trees, obviously the situation will change.

\section{Conclusions}

The applications of the drones in the field of agricultural curtains aim at the following problems:

A1) General inspection of protection curtains for proper maintenance;

A2) Estimating the effectiveness of protection curtains according to the season and the type of vegetation used;

A3) Surveillance of the effect of forest protection curtains on protected crops (the effect of uneven baking in the vicinity of the curtain, harvest losses, humidity, exposure to light and temperature, etc.);

A4) Research activities on the relationship between optical porosity and dynamic porosity, depending on the type of vegetation used, the wind speed, the season, the vegetation response to some pest attacks.

For more details, see the source [18].

\section{Acknowledgement}

This work was supported by a grant of the Romanian Research and Innovation Ministry, through Programme 1 - Development of the national research-development system, subprogram 1.2 Institutional performance - Projects for financing excellence in RDI, contract no. 16PFE, and by a grant of the Romanian Minister of Research and Innovation, CCCDI - UEFISCDI, project number PN-III- 
P1-1.2-PCCDI-2017-0560 "Eco-nanotechnologies and intelligent equipment for soil properties mapping and evaluating the dynamics of the plant in order to improve agricultural production and environmental protection", within PNCDI III.

\section{References}

1. Meriam - Webster dictionary, [Online]. Available: https://www.merriamwebster.com/dictionary/windbreak , (2018)

2. wikipedialwindbreaks, 17 July 2018. [Online]. Available: https://en.wikipedia.org/wiki/Windbreak , (2018)

3. H.B. Gonzales, M.E. Casada, L.J. Hagen, J. Tatarko, R.G. Maghiarang, C.J. Barden, Porosity and drag determination of a single-row vegetative barrier, Plant, Animal, \&Facility Systems Community of ASABE, 2017, (2017)

4. C. G. Bates, WINDBREAKS: Their influence and value, Washington: Government Printing Office, (1911)

5. I. Musat, Gh. Guiman, Perdelele forestiere - modalitate eficienta de protectie a cailor de comunicatie si transport, Bucuresti: Academia Romana, www.acad.ro/com2014/doc/d0515/08Musat_Ilie.pps, (2014)

6. Wind-Sensors/Products/FT205, [Online]. Available: https://fttechnologies.com/WindSensors/Products/FT205, (2018)

7. Applications/Applications/Drones-and-UAVs, [Online]. Available: https://fttechnologies.com/Applications/Applications/Drones-and-UAVs, (2018)

8. G. Giebel, U. Schmidt Paulsen, J. Bange, A. la Cour-Harbo, J. Reuder, S. Mayer, J. Mølgaard, Autonomous Aerial Sensors for Wind Power Meteorology - A Pre-Project Danmarks Tekniske Universitet, Risø Nationallaboratoriet for Bæredygtig Energi. Denmark. Forskningscenter Risoe. Risoe-R, (2012)

9. Applied Technologies, Inc., 2016. [Online]. Available: http://www.apptech.com/products/ultrasonic-anemometers/trisonica-mini/ , (2016)

10. Gh. D. Ionescu, Introducere in hidraulica, Bucuresti: Editura tehnica, (1977)

11. J.R. Brandle, Sh. Finch, "EC91-1763-B How Windbreaks Work, University of Nebraska, Lincoln, (1991)

12. M. Rosculet, Analiza Matematica, Bucuresti: Editura Didactica si Pedagogica, (1984)

13. Kika de la Garza Plant Materials Center, Vegetative Barriers For Erosion Control, Texas State Soil and Water Conservation Board, United States Environmental Protection Agency, Texas, (2013)

14. D. Rehacek, T. Khel, J. Kucera, J. Vopravil, M. Petera, Effect of Windbreaks on Wind Speed Reduction and Soil Protection against Wind Erosion, Soil \& Water Res., (2016)

15. H.B. Gonzales, Aerodynamics of wind erosion and particle collection through vegetative controls, Manhattan, Kansas: Kansas State University, (2015)

16. G. Li, R.G. Maghirang, Numerical Simulation of Airflow and Particle Collection by Vegetative Barriers, Engineering Applications of Computational Fluid Mechanics Vol. 6, No.1, p. 110-122, (2012)

17. "GEONICA". [Online]. Available: http://english.geonica.com/prod/79/137/WindSpeed-\&-Direction/index.html. (2018)

18. P. Cardei, Drones and windbreaks, preprint, DOI: 10.13140/RG.2.2.15716.83843, https://www.researchgate.net/publication/329356139_Drones_and_windbreaks 